\title{
Low serum albumin as a risk factor for infection-related in-hospital death among hemodialysis patients hospitalized on suspicion of infectious disease: a Japanese multicenter retrospective cohort study
}

\author{
Shun Minatoguchi ${ }^{1,2^{*}}$ (D) Atsushi Nomura ${ }^{1}$, Takahiro Imaizumi ${ }^{1,2}$, Sho Sasaki ${ }^{3}$, Takaya Ozeki ${ }^{2,4}$, Daisuke Uchida ${ }^{5}$,
} Hiroo Kawarazaki ${ }^{6}$, Fumihiko Sasai ${ }^{7}$, Keigo Tomita ${ }^{8}$, Hideaki Shimizu ${ }^{1}$ and Yoshiro Fujita ${ }^{1}$

\begin{abstract}
Background: Serum albumin is a marker of nourishment and inflammation. Although hypoalbuminemia in hemodialysis patients is reported as a risk factor for poor prognosis, few studies describe its effects on infectious diseases specifically. This study aimed to examine the relationship between the serum albumin level on admission and infection-related in-hospital death among hemodialysis patients.

Methods: This was a multicenter retrospective observational study that was undertaken in Japan. We reviewed the medical records of 507 hemodialysis patients aged $>18$ years, whose blood cultures were obtained based on suspicion of infectious disease, and who were managed at seven Japanese tertiary dialysis units from August 2011 to July 2013. The outcome measure was infection-related in-hospital death. Multivariate logistic regression models adjusted for age, sex, the dialysis vintage, diabetes mellitus, bacteremia, and log C-reactive protein levels were used for the statistical analysis.

Results: Four hundred patients were analyzed and allocated to three groups based on their serum albumin levels: marked hypoalbuminemia $(<2.5 \mathrm{mg} / \mathrm{dL})$, mild hypoalbuminemia $(\leq 2.5-<3.5 \mathrm{mg} / \mathrm{dL})$, and normal albumin levels $(\leq 3.5 \mathrm{mg} / \mathrm{dL})$. The infection-related in-hospital death rates were $22.9 \%(n=11), 12.5 \%(n=25)$, and $4.6 \%(n=7)$, respectively. The multivariate logistic regression models determined that a low serum albumin level was an independent risk factor for infection-related in-hospital death (odds ratio 0.35 , 95\% confidence interval 0.18-0.66).

Conclusions: A low serum albumin level strongly predicts infection-related in-hospital death in hemodialysis patients hospitalized on suspicion of infection. Like those with bacteremia or diabetes mellitus, hemodialysis patients with hypoalbuminemia require careful management of their infections.
\end{abstract}

Keywords: Albumin, Dialysis, Hypoalbuminemia, Infection, Mortality

\footnotetext{
* Correspondence: shunminatoguchi@gmail.com

${ }^{1}$ Department of Nephrology, Chubu Rosai Hospital, Nagoya, Japan

${ }^{2}$ Department of Nephrology, Nagoya University Graduate School of Medicine,

65 Tsurumai-cho, Showa-ku, Nagoya 466-8550, Japan

Full list of author information is available at the end of the article
}

(c) The Author(s). 2018 Open Access This article is distributed under the terms of the Creative Commons Attribution 4.0 International License (http://creativecommons.org/licenses/by/4.0/), which permits unrestricted use, distribution, and reproduction in any medium, provided you give appropriate credit to the original author(s) and the source, provide a link to the Creative Commons license, and indicate if changes were made. The Creative Commons Public Domain Dedication waiver (http://creativecommons.org/publicdomain/zero/1.0/) applies to the data made available in this article, unless otherwise stated. 


\section{Background}

Infectious disease is one of the most important causes of morbidity and mortality among patients with end-stage renal disease (ESRD) [1-3]. According to the United States Renal Data System, infection is the second leading cause of death among patients with ESRD, and it comprises almost 9\% of all deaths [4]. Similarly, almost 6000 hemodialysis patients in Japan die of infectious diseases annually, which amounts to approximately $20 \%$ of all-cause mortality of hemodialysis patients, and this rate is increasing every year $[5,6]$. Given the high prevalence of infectious disease, early predictions of infection-related mortality could improve the outcome for hemodialysis patients.

Reports from previous studies have described diabetes, cancer, multisystem disease, vascular access type, serum albumin level, and being female as risk factors for infection-related mortality in patients who undergo renal replacement therapy [1, 7-9]. In this study, our focus was on serum albumin levels. Serum albumin is a known marker of nourishment, and it is also recognized as a marker of inflammation because it is an acute phase reactant [10-12]. Hypoalbuminemia in hemodialysis patients has been described as a risk factor for poor prognosis such as all-cause and cardiovascular-related mortality [8, 13-17]. However, infection-related mortality has been discussed in only two articles. Wang et al. [17] showed an association between serum albumin levels and bacteremia-related mortality, but did not discuss non-bacteremic infectious disease. Mehrotra et al. [8] discussed baseline and time-averaged serum albumin levels and changes in serum albumin levels, but they did not mention serum albumin levels at the onset of infectious disease.

In the general population, hypoalbuminemia has been described as a risk factor that is associated with poor clinical outcomes in acute illness [18], and it has been reported that the serum albumin level on admission is an independent risk factor associated with death, length of stay, and re-admission [19].

Although demonstrated in the general population, there has been insufficient evidence to support an association between hypoalbuminemia on admission and short-term mortality among hemodialysis patients who are usually more prone to develop hypoalbuminemia than the general population [10].

Therefore, we hypothesized that a low serum albumin level on admission is an important prognostic factor that is associated with short-term mortality, such as infection-related in-hospital death, among hemodialysis patients, as is the case within the general population. This study aimed to examine the influence of the serum albumin level on infection-related in-hospital death among chronic dialysis patients from whom blood cultures were obtained and who were hospitalized on suspicion of infectious disease.

\section{Methods \\ Study population}

This was a Japanese, multicenter, retrospective, observational study that involved hemodialysis patients who were managed at seven Japanese tertiary dialysis units from August 2011 to July 2013. We reviewed the medical records of consecutive hemodialysis patients who were over 18 years of age and from whom blood cultures were obtained at least twice based on the suspicion of infectious disease in an outpatient setting or within $48 \mathrm{~h}$ after admission. The dialysis units involved were participants in the Japanese Investigators with Innovative Network about Kidney Disease group (JOINT-KD group), which includes Chubu Rosai Hospital, Toyohashi Municipal Hospital, St. Marianna University School of Medicine Hospital, Kawasaki Municipal Tama Hospital, Inagi Municipal Hospital, Showa University Fujigaoka Hospital, and Iizuka Hospital. A total of 507 patients who were undergoing hemodialysis were enrolled to participate in this study. Patients who were not hospitalized or whose serum albumin level data were missing were excluded from the study.

\section{Data collection}

The patients' demographic, clinical, and laboratory data were extracted from their medical records. The laboratory data, including the serum albumin levels, were extracted from the results of the blood tests that were undertaken on admission.

\section{Outcome measures}

The study's primary outcome measure was infection-related in-hospital death. The definition of infection-related in-hospital death was death during hospitalization with a final diagnosis of an infectious disease. The final diagnoses were determined by the attending physicians in each hospital.

\section{Statistical analyses}

We allocated the patients to three groups based on their serum albumin levels: marked hypoalbuminemia (group $1<2.5 \mathrm{mg} / \mathrm{dL}$ ), mild hypoalbuminemia (group $2 \leq 2.5-<3.5 \mathrm{mg} / \mathrm{dL}$ ), and normal albumin levels (group $3 \leq 3.5 \mathrm{mg} / \mathrm{dL}$ ) and then the groups were evaluated by their baseline characteristics. The cutoff values were based on those of a previous study of hypoalbuminemia [20]. The continuous variables are expressed as the medians and interquartile ranges, and the categorical variables are expressed as numbers and percentages. 
A logistic regression model was used to calculate the odds ratios (ORs) for infection-related in-hospital death, using group 3 as the reference category. This model was adjusted for age, sex, the dialysis vintage, diabetes mellitus, and bacteremia. These covariates were selected based on previous reports that describe the risk factors for infection-related mortality among hemodialysis patients $[1,21,22]$. We also calculated the ORs using the serum albumin levels as continuous variables in four models. Model 1 was unadjusted; model 2 was adjusted for age, sex, and present bacteremia; model 3 was adjusted for age, sex, present bacteremia, diabetes mellitus, and the dialysis vintage; and model 4 was adjusted for age, sex, present bacteremia, diabetes mellitus, the dialysis vintage, and the logarithmic $(\log ) \mathrm{C}$-reactive protein (CRP) levels. A log transformation was applied to the CRP levels because of positive skewness (1.5). Multicollinearity among all of the covariates was evaluated using the variance inflation factor (VIF). A VIF $>10$ indicates serious multicollinearity, and VIF values $>4$ may be causes for concern.

In addition, we carried out a sensitivity analysis. We calculated the ORs using logistic regression models that incorporated all of the in-hospital cases, which included the cases for whom the serum albumin data were missing. The cases in which serum albumin data were missing were included after multiple imputation.

All of the analyses, with the exception of the sensitivity analysis, were performed using IBM SPSS Statistics, version 24.0 (IBM Corporation, Armonk, NY, USA). The sensitivity analysis was performed using $\mathrm{R}$ software, version 3.3.1 (The Comprehensive $\mathrm{R}$ Archive Network: https://cran.r-project.org). The multiple imputation was performed using the "mice" package [23]. A $P$ value of $<0.05$ was considered statistically significant.

\section{Results}

Baseline characteristics

A total of 507 hemodialysis patients were included in this study, and 400 patients were analyzed after excluding the patients who were not hospitalized and those whose serum albumin data were missing (Fig. 1). Table 1 shows the patients' baseline characteristics categorized according to the serum albumin groups. Mean age was 74 years. Table 2 presents the outcomes after admission, including bacteremia, infectious diseases, and mortality. The proportions of hemodialysis patients with final infectious disease diagnoses were $68.8 \%(n=33), 62.0 \%$ $(n=124)$, and $56.6 \%(n=86)$, in group 1 , group 2 , and group 3, respectively. The infection-related in-hospital death rates were $22.9 \%(n=11), 12.5 \%(n=25)$, and $4.6 \%$ $(n=7)$, and the proportions of patients with bacteremia were $18.8 \%(n=9), 16.5 \%(n=33)$, and $13.8 \%(n=21)$ in group 1 , group 2 , and group 3 , respectively.

\section{Multivariate analysis of the risk factors for infection-related in-hospital death}

Figure 2 presents the logistic regression model of infection-related in-hospital deaths that used group 3 as the reference category. The ORs for infection-related in-hospital deaths were 8.17 (95\% CI 2.45-29.96) for group 1 and 3.03 (95\% CI 1.13-8.12) for group 2. The logistic regression models in which the serum albumin levels were used as continuous variables are shown in Tables 3 and 4. The serum albumin level, diabetes mellitus, and bacteremia were significant risk factors for poor clinical outcomes in hemodialysis patients (Table 3). The four models used to analyze the serum albumin levels (Table 4) all showed significant associations between the serum albumin level on admission and infection-related in-hospital death. We assessed the covariates'

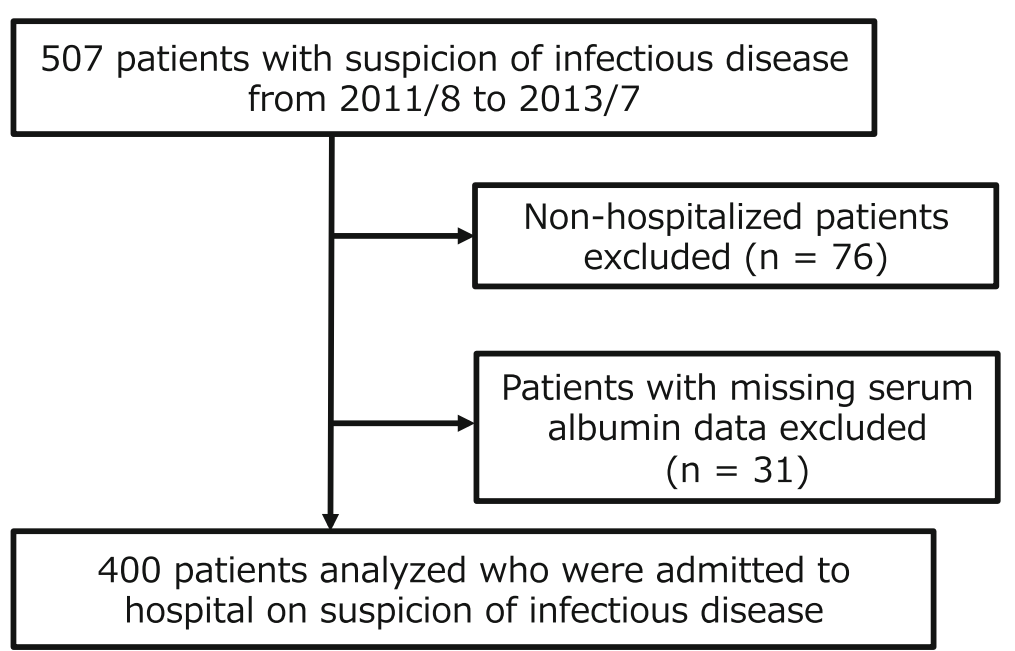

Fig. 1 Study flow chart 
Table 1 Baseline characteristics $(N=400)$

\begin{tabular}{|c|c|c|c|c|c|}
\hline & Alb $<2.5 n=48$ & $2.5 \leq$ Alb $<3.5 n=200$ & $3.5 \leq$ Alb $n=152$ & Total $n=400$ & $P$ value \\
\hline Albumin (g/dL) & $2.2(2.0,2.4)$ & $3.1(2.9,3.3)$ & $3.8(3.6,4.0)$ & $3.3(2.9,3.7)$ & $<0.01^{*}$ \\
\hline Age (years) & $72(66,80)$ & $75(69,81)$ & $71(62,79)$ & $74(66,81)$ & $<0.01^{*}$ \\
\hline Sex (male) & $25(52.1)$ & $125(62.5)$ & $110(72.4)$ & $260(65.0)$ & $0.021^{*}$ \\
\hline Dialysis vintage (month) & $54(25,130)$ & $63(24,119)$ & $62(24,108)$ & $62(24,116)$ & 0.924 \\
\hline BMI $\left(\mathrm{kg} / \mathrm{m}^{2}\right)$ & $19.7(17.2,21.6)$ & $19.5(17.5,22.0)$ & $21.5(19.0,23.7)$ & $20.1(17.9,22.8)$ & $<0.01^{*}$ \\
\hline Diabetes mellitus & $19(40.4)$ & $84(42.0)$ & $74(48.7)$ & $177(44.4)$ & 0.387 \\
\hline Malignancy & $7(14.6)$ & $27(13.5)$ & $16(10.5)$ & $50(12.5)$ & 0.633 \\
\hline Artificial devices & $10(20.8)$ & 77 (38.5) & $36(23.7)$ & $123(30.8)$ & $<0.01^{*}$ \\
\hline Creatinine (mg/dL) & $4.8(4.0,6.9)$ & $6.2(4.5,8.3)$ & $7.3(5.4,9.8)$ & $6.4(4.6,8.6)$ & $<0.01^{*}$ \\
\hline Potassium (mEq/L) & $3.8(3.4,4.4)$ & $4.3(3.7,4.8)$ & $4.3(4.1,5.2)$ & $4.3(3.8,4.8)$ & $<0.01^{*}$ \\
\hline CRP (mg/dL) & $10.1(4.5,21.5)$ & $7.7(3.4,14.4)$ & $4.0(0.7,9.7)$ & $6.6(1.9,12.9)$ & $<0.01^{*}$ \\
\hline Hemoglobin (g/dL) & $9.7(8.4,11.5)$ & $10.6(9.5,11.5)$ & $11.3(10.2,12.4)$ & $10.7(9.5,11.9)$ & $<0.01^{*}$ \\
\hline
\end{tabular}

BMI body mass index; CRP C-reactive protein

Continuous data are medians (IQR). Categorical data are $n$ values (\%)

Differences among groups were evaluated by a chi-square test for categorical variables and a Kruskall-Wallis test for continuous variables

$* P<0.05$

multicollinearity using the VIF in this analysis, and all of the VIFs were $<4.0$.

Table 5 shows the results of the sensitivity analysis that comprised multivariate logistic regression analysis after multiple imputation, which determined that a low serum albumin level was an independent risk factor for infection-related in-hospital death, consistent with the results from the multivariate analysis without multiple imputation.

\section{Discussion}

The results from the present study showed that the serum albumin level on admission was a significant risk factor for in-hospital infection-related death among hemodialysis patients from whom blood cultures were obtained and who had been hospitalized on suspicion of infectious disease. Hemodialysis patients who are hospitalized with infectious diseases have high infection-related mortality and readmission rates [24].
Indeed, infection-related mortality is significantly higher in ESRD patients with bacteremia compared with patients without bacteremia [21]. In our study, the bacteremia-related in-hospital death rate was $6.3 \%$, which represented $58.1 \%$ of all of the infection-related in-hospital deaths. However, mortality in patients with infectious diseases in the absence of bacteremia is not negligible. Dalrymple et al. [24] reported that the in-hospital mortality rates for infection-related hospitalization without bloodstream infections or sepsis were between 2 and 16\%, which concurs with the findings from our study that showed that the rate of infection-related in-hospital death without bacteremia was $4.5 \%$. In addition, although there is a strong association between bacteremia and poor clinical outcomes, it is difficult to determine whether or not patients have bacteremia on admission. In contrast, the serum albumin levels are assessable on admission; hence, our study's findings suggest that the serum albumin levels

Table 2 Outcomes after admissions $(N=400)$

\begin{tabular}{|c|c|c|c|c|c|}
\hline & Alb $<2.5 n=48$ & $2.5 \leq \mathrm{Alb}<3.5 n=200$ & $3.5 \leq$ Alb $n=152$ & Total $n=400$ & $P$ value \\
\hline Bacteremia & $9(18.8)$ & $33(16.5)$ & $21(13.8)$ & $63(15.8)$ & 0.657 \\
\hline Final diagnosis of infectious disease & $33(68.8)$ & $124(62.0)$ & $86(56.6)$ & $243(60.8)$ & 0.282 \\
\hline Duration of hospitalization (days) & $22(9,52)$ & $19(11,37)$ & $13(7,25)$ & $16(9,35)$ & $<0.01^{*}$ \\
\hline In-hospital death (infection related) & $11(22.9)$ & $25(12.5)$ & $7(4.6)$ & $43(10.8)$ & $<0.01^{*}$ \\
\hline Duration from admission to infection-related death (days) & $9(5,29)$ & $15(6,25)$ & $11(2,45)$ & $14(6,28)$ & 0.913 \\
\hline In-hospital death (infection related, with bacteremia) & $5(10.4)$ & $15(7.5)$ & $5(3.3)$ & $25(6.3)$ & 0.121 \\
\hline In-hospital death (infection related, without bacteremia) & $6(12.5)$ & $10(5.0)$ & $2(1.3)$ & $18(4.5)$ & $<0.01^{*}$ \\
\hline In-hospital death (all cause) & $15(31.3)$ & $40(20.0)$ & $12(7.9)$ & $67(16.8)$ & $<0.01^{*}$ \\
\hline
\end{tabular}

Continuous data are medians (IQR). Categorical data are $\mathrm{n}$ values (\%)

Differences among groups were evaluated by a chi-square test for categorical variables and a Kruskall-Wallis test for continuous variables ${ }^{*} P<0.05$ 


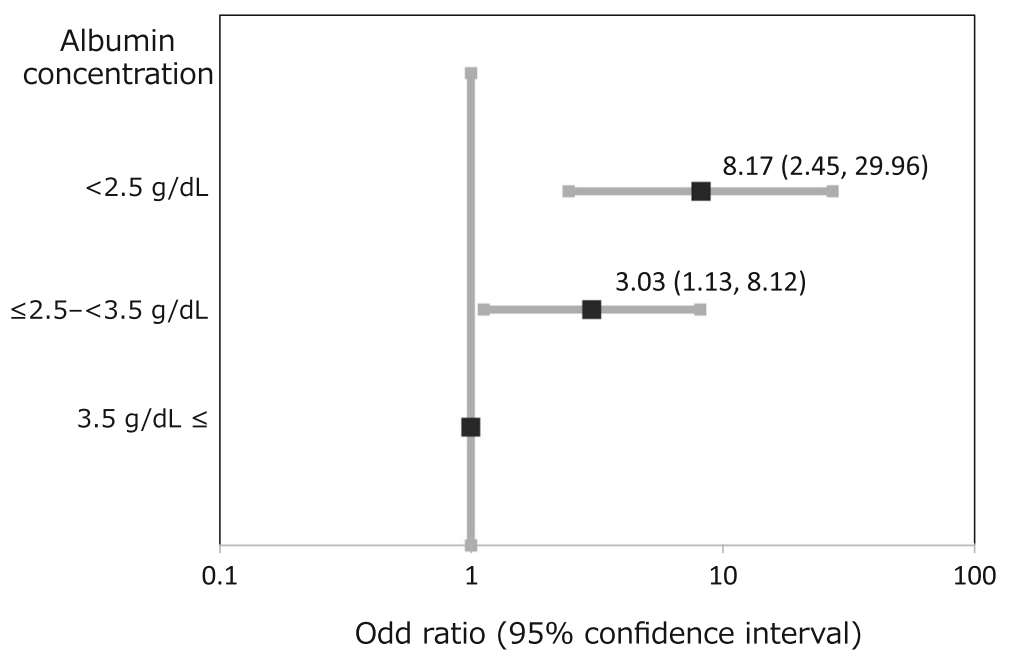

Fig. 2 Adjusted odds ratios (95\% confidence intervals) for infection-related in-hospital death according to the albumin groups. The odds ratios were adjusted simultaneously for age, sex, the dialysis vintage, diabetes mellitus, and current bacteremia. Alb, albumin

may provide earlier prognostic predictions among patients who are suspected of carrying infectious diseases.

There are several strengths associated with our study that include its multicenter design. Several studies have assessed the relationship between hemodialysis and serum albumin levels, but few multicenter cohort studies that have specifically investigated infectious diseases among hemodialysis patients have been undertaken. This study focused on infectious diseases specifically, which enabled us to assess the data that were derived from the onset of the infectious diseases and their effects on the patients' prognoses. Additionally, the mean age of this cohort, which was 74 years, was important. The mean age in previous studies that assessed the relationship between hypoalbuminemia and infection-related mortalities were around 60 years $[8,17]$. This is not applicable to the increased age of dialysis patients in recent years [5]. Although it has been shown that elderly patients are prone to hypoalbuminemia [25], we suggest that hypoalbuminemia is still a strong prognostic factor of infectious disease in elderly dialysis patients.

Our study has some limitations. First, the study's sample size was small compared with previous studies. This might have lowered our study's statistical power, but the findings from the multivariate analysis suggested a strong association between a low serum albumin level and poor outcomes. In addition, this was a multicenter study; hence, its external validity was high. The infection-related in-hospital death rate in our study was $10.8 \%$, which concurs with that reported previously [24]. Second, there were no data that described the patients' courses after discharge and there was no information

Table 3 Univariate and multivariate logistic regression analysis of in-hospital infection related death

\begin{tabular}{|c|c|c|c|c|c|c|}
\hline & \multicolumn{3}{|c|}{ Univariate analysis } & \multicolumn{3}{|c|}{ Multivariate analysis" } \\
\hline & $\overline{\mathrm{OR}}$ & {$[95 \% \mathrm{Cl}]$} & $P$ & $\overline{\mathrm{OR}}$ & {$[95 \% \mathrm{Cl}]$} & $P$ \\
\hline Sex (reference: female) & 1.27 & {$[0.64,2.53]$} & 0.49 & 1.68 & {$[0.74,3.83]$} & 0.22 \\
\hline Age (per year increase) & 1.01 & {$[0.98,1.04]$} & 0.42 & 1.01 & {$[0.97,1.05]$} & 0.53 \\
\hline $\begin{array}{l}\text { Dialysis vintage } \\
\text { (per month increase) }\end{array}$ & 1.00 & {$[1.00,1.01]$} & 0.09 & 1.00 & {$[1.00,1.01]$} & 0.14 \\
\hline $\begin{array}{l}\text { Diabetes mellitus } \\
\text { (reference: without DM) }\end{array}$ & 1.22 & {$[0.65,2.31]$} & 0.53 & 2.35 & {$[1.03,5.35]$} & $0.041^{*}$ \\
\hline $\begin{array}{l}\text { Bacteremia } \\
\text { (reference: without bacteremia) }\end{array}$ & 11.66 & {$[5.83,23.32]$} & $<0.01^{*}$ & 11.47 & {$[5.04,26.01]$} & $<0.01^{*}$ \\
\hline $\begin{array}{l}\log \text { CRP } \\
\text { (per point increase) }\end{array}$ & 2.04 & {$[1.44,2.90]$} & $<0.01^{*}$ & 1.36 & {$[0.94,1.99]$} & 0.11 \\
\hline $\begin{array}{l}\text { Serum albumin } \\
\text { (per g/dl increase) }\end{array}$ & 0.38 & {$[0.23,0.63]$} & $<0.01^{*}$ & 0.35 & {$[0.18,0.66]$} & $<0.01^{*}$ \\
\hline
\end{tabular}

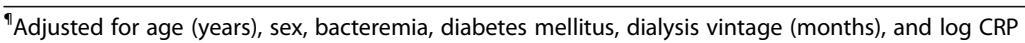
$* P<0.05$ 
Table 4 Odd's ratio for association of serum albumin (per $\mathrm{g} / \mathrm{dl}$ increase) with infection related in-hospital death

\begin{tabular}{llll}
\hline Model & OR & {$[95 \% \mathrm{Cl}]$} & $P$ \\
\hline $1^{\mathrm{a}}$ & 0.38 & {$[0.23,0.63]$} & $<0.01^{*}$ \\
$2^{\mathrm{b}}$ & 0.32 & {$[0.18,0.57]$} & $<0.01^{*}$ \\
$3^{\mathrm{c}}$ & 0.30 & {$[0.16,0.55]$} & $<0.01^{*}$ \\
$4^{\mathrm{d}}$ & 0.35 & {$[0.18,0.66]$} & $<0.01^{*}$ \\
\hline
\end{tabular}

anadjusted

${ }^{\mathrm{b}}$ Adjusted for age (years), sex, and bacteremia

'Adjusted for age (years), sex, bacteremia, diabetes mellitus, and

dialysis vintage(months)

${ }^{\mathrm{d}}$ Adjusted for age (years), sex, bacteremia, diabetes mellitus, dialysis vintage (months), and log CRP

${ }^{*} P<0.05$

regarding the patients' courses when they changed hospitals. In this study, 67 patients died during hospitalization, 265 patients were discharged from hospital, 64 patients were transferred to different hospitals, and 4 patients' courses were unknown. There were no data that described the mortality of the patients who were transferred. Third, the inclusion criteria were not very clear. Suspicion of infectious disease was subjectively assessed by each physician thereby creating a sampling bias. However, this does reflect the actual clinical management of dialysis patients. Fourth, we did not have data regarding other malnutrition and inflammation markers such as prealbumin, cholesterol, other rapid turnover proteins, and liver enzymes. Although low albumin levels are linked to a poor prognosis in this study, reasons for the hypoalbuminemia were not clear. There are many factors that affect serum albumin levels and our data showed that body mass index (BMI), CRP, and creatinine levels in the three albumin groups were statistically different. This means that the reasons for hypoalbuminemia in each case could be different. We could not estimate how malnutrition and inflammation impacted hypoalbuminemia due to the lack of data

Table 5 Sensitivity analysis: multivariate logistic regression analysis of in-hospital infection-related death after multiple imputation

\begin{tabular}{llll}
\hline & \multicolumn{4}{l}{$\begin{array}{l}\text { Multivariate analysis } \\
\text { (Ml data) }\end{array}$} \\
\cline { 2 - 4 } & OR & {$[95 \% \mathrm{Cl}]$} & $P$ \\
\hline Sex (reference: female) & 1.68 & {$[0.75,3.75]$} & 0.20 \\
Age (per year increase) & 1.02 & {$[0.98,1.06]$} & 0.38 \\
Dialysis vintage (per month increase) & 1.00 & {$[1.00,1.01]$} & 0.14 \\
Diabetes mellitus (reference: without DM) & 2.16 & {$[0.98,4.73]$} & 0.06 \\
Bacteremia (reference: without bacteremia) & 10.88 & {$[4.91,24.11]$} & $<0.01^{*}$ \\
log CRP (per point increase) & 1.42 & {$[0.98,2.07]$} & 0.07 \\
Serum albumin (per g/dl increase) & 0.34 & {$[0.18,0.64]$} & $<0.01^{*}$ \\
\hline Adjustd for age &
\end{tabular}

Adjusted for age (years), sex, bacteremia, diabetes mellitus, dialysis vintage (months), and log CRP

${ }^{*} P<0.05$ regarding other more specific markers, which could be a limitation. However, serum albumin levels are commonly assessed by clinicians and are very useful regardless of what causes the hypoalbuminemia. Fifth, as mentioned above, CRP levels in group 1 were negligible. This suggests high CRP levels are linked to poor prognosis, and the OR in the logistic regression model with CRP levels (Table 4, model 4) increased compared with the model without CRP levels (Table 4, model 3). The effect of hypoalbuminemia was attenuated by CRP due to the common causal pathway of inflammation. However, CRP has not been identified as a prognosis factor for infectious disease in past studies despite its predictive association with bacteremia and elevated inflammatory conditions [26, 27]. Finally, the timing of the serum albumin measurements was an important limitation. The serum albumin levels were only measured on admission in this study. Although we discussed serum albumin levels as an acute inflammatory reactant rather than a nutrient marker, single measurement of albumin levels was not enough to judge whether the hypoalbuminemia was chronic, whether it had occurred as a result of acute illness, or both. So, we assessed the multicollinearity between serum albumin levels and BMI, a known nutritional marker, and the VIF was < 4.0. This suggests that hypoalbuminemia in this study reflected not only malnutrition, which could be chronic hypoalbuminemia, but also acute reaction from inflammation. We did not evaluate the nutritional status of the participants other than BMI, so this could be a limitation. Furthermore, the serum albumin levels of hemodialysis patients fluctuate depending on the volume status; therefore, compared with the pre-dialysis levels, the post-dialysis serum albumin levels are higher, and the degrees of fluctuation correlate with the intra-dialysis volume loss. Kubrusly et al. [28] reported a comparative analysis of the pre- and post-dialysis albumin levels, and the mean albumin levels were $3.45 \pm 0.55 \mathrm{~g} / \mathrm{dL}$ and $3.90 \pm 0.73 \mathrm{~g} / \mathrm{dL}$, respectively. Although these findings indicated that the intra-dialysis albumin fluctuations are not large, the timing of the serum albumin measurements could be a study limitation as we did not have any data that described the relationship between the timing of the blood tests and dialysis.

\section{Conclusions}

The present study's findings showed that the serum albumin level was a significant risk factor for infection-related in-hospital death among hemodialysis patients who had been hospitalized on suspicion of infectious disease. Like those with bacteremia or diabetes mellitus, hemodialysis patients with hypoalbuminemia and infections should be carefully managed. 


\section{Abbreviations}

BMl: Body mass index; CRP: C-reactive protein; ESRD: End-stage renal disease; ORs: Odds ratios; VIF: Variance inflation factor

\section{Acknowledgements}

The authors would particularly like to thank Dr. Yugo Shibagaki (St. Marianna University School of Medicine Hospital, Kawasaki, Japan) for the helpful discussions. We are also grateful for the time and efforts of the nephrologists, clinical research assistants, and patients of St. Marianna University School of Medicine, Toyohashi Municipal Hospital, Inagi Municipal Hospital, Kawasaki Municipal Tama Hospital, St. Marianna University School of Medicine Hospital, Showa University Fujigaoka Hospital, lizuka Hospital, and Chubu Rosai Hospital.

\section{Availability of data and materials}

The datasets of the current study is available from the corresponding author on a reasonable request.

\section{Authors' contributions}

SM and AN designed the study. SM performed the statistical analysis and wrote the manuscript. All other authors contributed to the study design, data collection, analysis, and writing of the manuscript. All authors read and approved the final manuscript.

\section{Ethics approval and consent to participate}

The study was conducted with approval from the St. Marianna University School of Medicine's Ethics Committee (No. 2713), and it was conducted in accordance with the principles of the Declaration of Helsinki. Informed consent was not obtained from the participants, because of the retrospective nature of the study.

\section{Consent for publication}

Not applicable

\section{Competing interests}

The authors declare that they have no competing interests.

\section{Publisher's Note}

Springer Nature remains neutral with regard to jurisdictional claims in published maps and institutional affiliations.

\section{Author details}

'Department of Nephrology, Chubu Rosai Hospital, Nagoya, Japan. 2Department of Nephrology, Nagoya University Graduate School of Medicine, 65 Tsurumai-cho, Showa-ku, Nagoya 466-8550, Japan. ${ }^{3}$ Division of Nephrology and Hypertension, St. Marianna University School of Medicine Hospital, Kawasaki, Japan. ${ }^{4}$ Department of Nephrology, Toyohashi Municipal Hospital, Toyohashi, Japan. ${ }^{5}$ Department of Nephrology and Hypertension, Kawasaki Municipal Tama Hospital, Kawasaki, Japan. ${ }^{6}$ Department of Nephrology, Inagi Municipal Hospital, Tokyo, Japan. ${ }^{7}$ Department of Nephrology, Showa University Fujigaoka Hospital, Yokohama, Japan. ${ }^{8}$ Department of Nephrology, lizuka Hospital, lizuka, Japan.

\section{Received: 16 February 2018 Accepted: 20 July 2018}

\section{Published online: 01 August 2018}

\section{References}

1. Vogelzang JL, van Stralen KJ, Noordzij M, Diez JA, Carrero JJ, Couchoud C, et al. Mortality from infections and malignancies in patients treated with renal replacement therapy: data from the ERA-EDTA registry. Nephrol Dial Transplant. 2015;30:1028-37.

2. Masakane I, Nakai S, Ogata S, Kimata N, Hanafusa N, Hamano T, et al. An overview of regular dialysis treatment in Japan (as of 31 December 2013). Ther Apher Dial. 2015;19:540-74.

3. Jaber BL. Bacterial infections in hemodialysis patients: pathogenesis and prevention. Kidney Int. 2005;67:2508-19.

4. Saran R, Li Y, Robinson B, Abbott KC, Agodoa LY, Ayanian J, et al. US Renal Data System 2015 Annual Data Report: epidemiology of kidney disease in the United States. Am J Kidney Dis. 2016;67:Svii-Sviii.

5. Masakane I, Nakai S, Ogata S, Kimata N, Hanafusa N, Hamano T, et al. Annual Dialysis Data Report 2014, JSDT Renal Data Registry (JRDR). Renal Replace Ther. 2017;3:1-43.
6. Wakasugi M, Kazama JJ, Narita I. Mortality trends among Japanese dialysis patients, 1988-2013: a joinpoint regression analysis. Nephrol Dial Transplant. 2016;31:1501-7.

7. Ros S, Remon C, Qureshi AR, Quiros P, Lindholm B, Carrero JJ. Increased risk of fatal infections in women starting peritoneal dialysis. Perit Dial Int. 2013; 33:487-94.

8. Mehrotra R, Duong U, Jiwakanon S, Kovesdy CP, Moran J, Kopple JD, et al. Serum albumin as a predictor of mortality in peritoneal dialysis: comparisons with hemodialysis. Am J Kidney Dis. 2011;58:418-28.

9. Dhingra RK, Young EW, Hulbert-Shearon TE, Leavey SF, Port FK. Type of vascular access and mortality in U.S. hemodialysis patients. Kidney Int. 2001; 60:1443-51

10. Friedman AN, Fadem SZ. Reassessment of albumin as a nutritional marker in kidney disease. J Am Soc Nephrol. 2010;21:223-30.

11. Kaysen GA, Stevenson FT, Depner TA. Determinants of albumin concentration in hemodialysis patients. Am J Kidney Dis. 1997;29:658-68.

12. Don BR, Kaysen G. Serum albumin: relationship to inflammation and nutrition. Semin Dial. 2004;17:432-7.

13. Owen WF, Lew NL, Liu Y, Lowrie EG, Lazarus JM. The urea reduction ratio and serum albumin concentration as predictors of mortality in patients undergoing hemodialysis. N Engl J Med. 1993;329:1001-6.

14. Kalantar-Zadeh K, Kilpatrick RD, Kuwae N, McAllister CJ, Alcorn H Jr, Kopple $J D$, et al. Revisiting mortality predictability of serum albumin in the dialysis population: time dependency, longitudinal changes and populationattributable fraction. Nephrol Dial Transplant. 2005;20:1880-8.

15. Honda H, Qureshi AR, Heimbürger $\mathrm{O}$, Barany $\mathrm{P}$, Wang $\mathrm{K}$, Pecoits-Filho $\mathrm{R}$, et al. Serum albumin, C-reactive protein, interleukin 6, and fetuin $A$ as predictors of malnutrition, cardiovascular disease, and mortality in patients with ESRD. Am J Kidney Dis. 2006;47:139-48.

16. Chiu P-F, Tsai C-C, Wu C-L, Yang T-Y, Liou H-H, Chen H-L, et al. Trajectories of serum albumin predict survival of peritoneal dialysis patients. Medicine. 2016;95:e3202.

17. Wang I-K, Chang Y-C, Liang C-C, Chuang F-R, Chang C-T, Lin H-H, et al. Bacteremia in hemodialysis and peritoneal dialysis patients. Intern Med. 2012:51:1015-21.

18. Vincent J-L, Dubois M-J, Navickis RJ, Wilkes MM. Hypoalbuminemia in acute illness: is there a rationale for intervention? Ann Surg. 2003:237:319-34.

19. Herrmann FR, Safran C, Levkoff SE, Minaker KL. Serum albumin level on admission as a predictor of death, length of stay, and readmission. Arch Intern Med. 1992;152:125-6.

20. Akirov A, Iraqi HM, Atamna A, Shimon I. Low albumin levels are associated with mortality risk in hospitalized patients. Am J Med. 2017;130:1465.e11-9.

21. Sarnak MJ, Jaber BL. Mortality caused by sepsis in patients with end-stage renal disease compared with the general population. Kidney Int. 2000;58: 1758-64.

22. Sumida K, Yamagata K, Iseki K, Tsubakihara Y. Different impact of hemodialysis vintage on cause-specific mortality in long-term hemodialysis patients. Nephrol Dial Transplant. 2016;31:298-305.

23. van Buuren S, Groothuis-Oudshoorn K. mice: Multivariate Imputation by Chained Equations in R. J Stat Softw. 2011:45:1-67.

24. Dalrymple LS, Mu Y, Romano PS, Nguyen DV, Chertow GM, Delgado C, et al. Outcomes of infection-related hospitalization in Medicare beneficiaries receiving in-center hemodialysis. Am J Kidney Dis. 2015;65:754-62.

25. Hanafusa N, Sakurai S, Nangaku M. Heterogeneity of clinical indices among the older dialysis population - a study on Japanese dialysis population. Renal Replac Ther. 2016;3:1-9.

26. Simon L, Gauvin F, Amre DK, Saint-Louis P, Lacroix J. Serum procalcitonin and C-reactive protein levels as markers of bacterial infection: a systematic review and meta-analysis. Clin Infect Dis. 2004;39:206-17. https://doi.org/10. 1086/421997

27. Hedlund J, Hansson LO. Procalcitonin and C-reactive protein levels in community-acquired pneumonia: correlation with etiology and prognosis. Infection. 2000;28:68-73.

28. Kubrusly M, de Oliveira CMC, de Oliveira Santos DC, Mota RS, Pereira ML. Comparative analysis of pre- and post-dialysis albumin levels as indicators of nutritional and morbidity and mortality risk in hemodialysis patients. J Bras Nefrol. 2012;34:27-35. 